\title{
A RARE CASE REPORT: MAPLE SYRUP URINE DISEASE
}

Suryakant Yashwant Ingale1, Prashant Popatlal Shah², Pratik Kamalaprasad Yadav³, Harshada Dilip Tatiya ${ }^{4}$, Raghunandan Reddy Bheem Reddy 5

${ }^{1}$ Associate Professor, Department of Paediatrics, Krishna Institute of Medical Sciences, Karad. ${ }^{2}$ Assistant Professor, Department of Paediatrics, Krishna Institute of Medical Sciences, Karad. ${ }^{3}$ Resident, Department of Paediatrics, Krishna Institute of Medical Sciences, Karad. ${ }^{4}$ Resident, Department of Paediatrics, Krishna Institute of Medical Sciences, Karad. ${ }^{5}$ Resident, Department of Paediatrics, Krishna Institute of Medical Sciences, Karad.

\section{ABSTRACT}

We report a case of Maple Syrup Urine Disease presenting in an 8-day-old neonate with classic MRI \& biochemical findings. MSUD is a rare autosomal recessive inborn error of metabolism. Its incidence is estimated to be around 1 in 185,000 infants worldwide. It is named because of its characteristic burnt sugar smell of urine. MSUD is caused by deficiency of BCKD leading to accumulation of BCAA and their toxic by-products in the blood and urine. Imaging is characterised by MSUD affecting myelinated white matter.

\section{KEYWORDS}

MSUD, Maple Syrup Urine Disease, Inborn Error of Metabolism, BCKD.

HOW TO CITE THIS ARTICLE: Ingale SY, Shah PP, Yadav PK, et al. A rare case report: Maple syrup urine disease. J. Evolution Med. Dent. Sci. 2016;5(81):6080-6082, DOI: 10.14260/jemds/2016/1373

\section{INTRODUCTION}

MSUD is an autosomal recessive disorder in newborn caused by deficiency of BCKD. Due to deficiency, there is abnormal oxidative decarboxylation of branch chain amino acids like leucine, isoleucine and valine. So there is accumulation of both amino acids and their corresponding alpha ketoacids in plasma and urine. Accumulation of leucine in particular causes neurological symptoms as it is rapidly transported across blood brain barrier. Elevation and accumulation of plasma isoleucine is associated with maple syrup odour. MSUD can be classified into 5 clinical phenotypes based on age of onset, severity of symptoms and response to thiamine treatment. Classic MSUD, Intermediate MSUD, Intermittent MSUD, Thiamine responsive MSUD, E-3/(Dihydrolipoyl dehydrogenase) MSUD deficient forms. CT, MRI, transcranial neurosonography show characteristic features of the disease. If not diagnosed and treated early, it progresses to increased intracranial pressure and death in few weeks.

\section{CASE REPORT}

An 8-day-old female baby presented with intractable seizures (Status epilepticus), tonic posturing, not accepting feeds, with mild grade fever. Moro's reflex was absent. Urine of baby had odour like burnt sugar (maple syrup). This was the fourth issue born by LSCS, $2^{\text {nd }}$ child of parents expired at the age of 1-1/2 month and $3^{\text {rd }}$ child at age of 16 days, both were male and both children were not investigated properly. $1^{\text {st }}$ issue is a 15-yearold female child, alive and healthy. Routine laboratory investigations were normal. CRP was negative. CSF examination was normal.

Financial or Other, Competing Interest: None.

Submission 01-09-2016, Peer Review 24-09-2016,

Acceptance 01-10-2016, Published 10-10-2016.

Corresponding Author:

Dr. Harshada Dilip Tatiya,

Room No. 305, IHR Hostel,

Krishna Institute of Medical Sciences,

Malkapur, Karad.

E-mail: harshadatatiya@hotmail.com

DOI: $10.14260 /$ jemds/2016/1373

\section{(c) $($ ) $९$}

Urine GC-MS (Gas chromatography - mass spectrometry) was done, which confirmed the diagnosis as Maple Syrup Urine Disease. Urine GC - MS showed elevation of MSUD metabolites (2-OH isovaleric, 2-Ketoisovaleric OX-2, 2-OH-3-methylvaleric2, 2-keto-3 methylvaleric OX-2, 2-ketoisocaproic OX-2). MRI showed multiple symmetrical areas of altered signal intensity in bilateral centrum semiovale, corona radiata, basal ganglia, thalamus, midbrain, pons, medulla, cerebellar peduncles and dentate nucleus of cerebellum, showing restriction of diffusion (Hyperintensity on DWI (Diffuse weighted MRI) and hypointense on ADC).

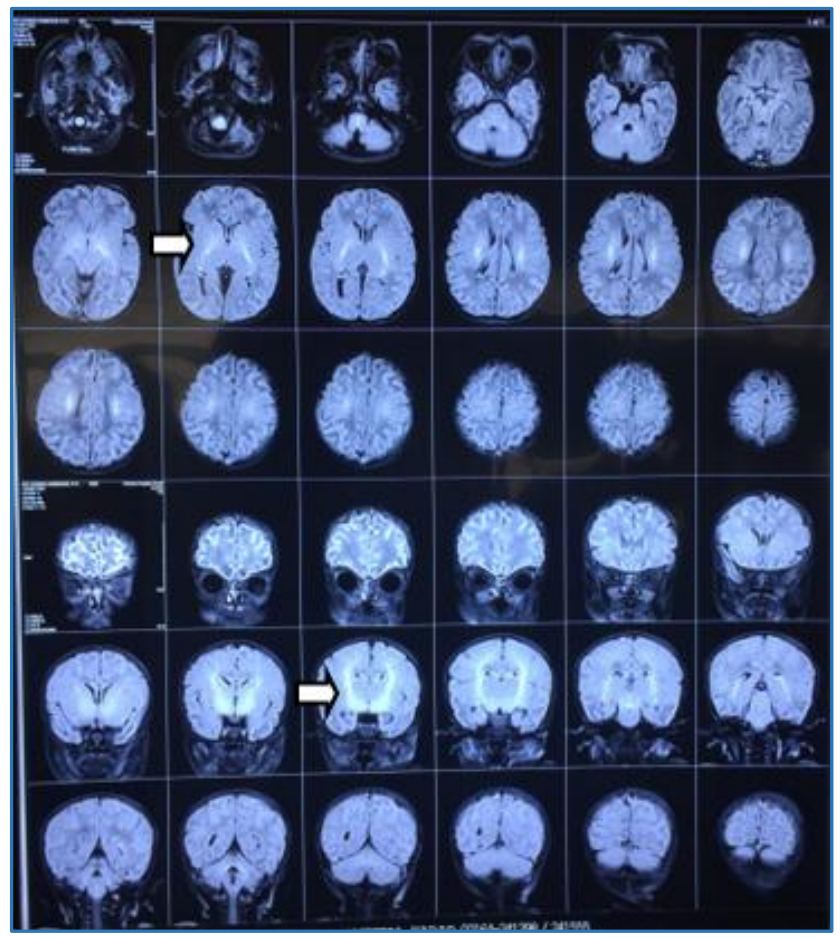



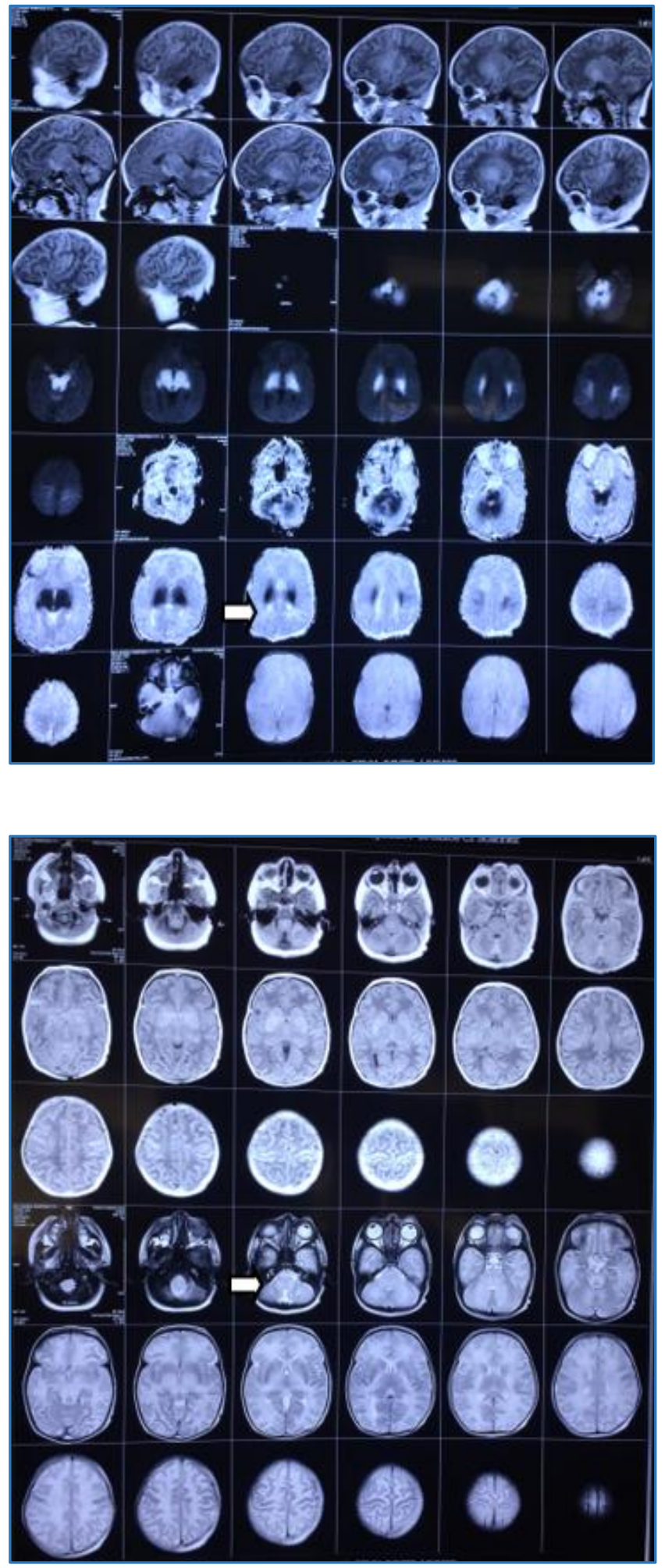

MRI films of the baby showing multiple symmetrical areas of altered signal intensity. Altered signal intensity is seen in bilateral centrum semiovale, corona radiata, basal ganglia, thalamus, midbrain, pons, medulla, cerebellar peduncles and dentate nucleus of cerebellum

Treatment given: Glucose Infusion, Midazolam drip, Inj. Ceftazidime, Inj. Vancomycin, Inj. Phenobarbitone, Inj. Fosphenytoin, Inj. Pyridoxine (Vitamin B6), Inj. Acyclovir, Syrup Carnisure (L-Carnitine), Tab. Zubitin (Biotin), Tab Methyl cobalamine (Vitamin B12), Inj. Thiamine (Vitamin B1), Capsule Ubi-Q (ubidecarenone).

\section{DISCUSSION}

History: First case was described by Menkes et al in 1954 after 4 Massachusetts siblings had died within first three months of their age due to a neurodegenerative disorder. The urine of these infants had an odour resembling maple syrup (Burned sugar).[1] Therefore, this disorder was called maple sugar urine disease and later maple syrup urine disease. Branched chain amino acid compounds and their corresponding alpha keto acids and the enzymatic defect in MSUD was demonstrated by Dancis et al.[2] Its incidence is estimated to be around 1 in 185,000 infants worldwide. ${ }^{[3]}$

\section{Types}

There are 5 distinct phenotypes based on age of onset, severity of symptoms and response to thiamine treatment. Severity is based on acute toxic phase.

\section{Classic MSUD}

Is the most common and most severe form of MSUD. Initial symptoms include poor feeding, vomiting, lethargy, poor weight gain. Symptoms develop in neonates aged 4-7 days. Breast-feeding may delay onset of symptoms to 2 nd week of life. In classic MSUD, neurological signs rapidly develop (Alternating muscular hypotonia and hypertonia, dystonia, seizures, encephalopathy). If untreated, can lead to significant developmental delay and babies die in 1 st month.

\section{Intermittent MSUD}

Is the 2nd most common form of MSUD. These patients develop normal growth and intelligence. These patients present during episodes of catabolic stress (Intermittent illness like otitis media, trauma, surgery). During episodes, patient may develop ataxia, lethargy, seizures and coma. If not treated appropriately, patients die.

\section{Intermediate MSUD}

Is much rarer. Clinical signs include neurological impairment, developmental delay of varying degree and seizures. It presents at any age, depending on residual BCKD activity.

\section{Thiamine Responsive MSUD}

Is a rare form of MSUD. Seriver et al reported the 1st case of thiamine responsive MSUD. After this case, other patients have been reported to show some improvement of metabolic control to thiamine along with dietary restriction of BCAA.

E-3 (dihydrolipoyl dehydrogenase) deficient form is a very rare form of MSUD. Clinical presentation is indistinguishable from intermediate MSUD with the exception of accompanying lactic acidosis. These patients have combined deficiency of BKCD, pyruvate and alpha ketoglutarate dehydrogenase complexes. Some researchers consider this a separate distinct disorder.

\section{Investigation and Workup}

Plasma amino acid levels to assess the elevation of BCAA and to detect alloisoleucine. Detection of alloisoleucine is diagnostic for MSUD. Alloisoleucine may not appear until the 6th day of life. BCAA may be elevated in patients with ketotic hypoglycaemia and in patients in post-absorptive state and in infants on TPN. 


\section{Urine Organic Acids}

Using GC-MS (Gas chromatography-mass spectrometry) to detect alpha-hydroxyisovalerate, lactate, pyruvate and alphaketoglutarate. Random urine sample is usually sufficient.

\section{Screening}

Is performed with tandem mass spectrometry using concentrations of leucine and isoleucine and Fisher ratio as diagnostic measures. Diagnosis should immediately be followed by treatment.

Molecular Testing is also available. It is preferred method for prenatal diagnosis. Neurosonography shows bilateral symmetrical increased echogenicity of periventricular white matter, thalami, basal ganglia. CT scan and MRI shows typical signs in form of localised oedema in brainstem, cerebellar white matter, cerebral peduncles, posterior limb of internal capsule, globus pallidus, perirolandic white matter. It may also show generalised oedema.[4]

In MSUD encephalopathy, 2 types of oedema are seen in MRI, intramyelinic oedema and vasogenic oedema. DWI is more useful as it detects both types of oedema. In diffuse weighted (D-W) MRI, It is hypothesised that in myelinated areas, hyperintensity was because of intramyelinic oedema; unmyelinated areas, hypointensity was because of vasogenic interstitial oedema.

\section{Treatment}

The two main aspects to the treatment of MSUD are long term management and treatment of episodes of acute metabolic decompensation. Mainstay of treatment is dietary restriction of BCAA.[5],[6],[7]

Episodes of metabolic decompensation should be treated aggressively. Initiate IV glucose infusions $(5-8 \mathrm{mg} / \mathrm{kg} / \mathrm{min}$.) for infants. Insulin infusions may be added to promote anabolism. Some patients have been responsive to thiamine. Thiamine can be administered in cases of thiamine responsiveness.

Long term management includes dietary \& surgical management. Dietary goal is normalisation of BCAA by restricting intake of BCAA. Dietary therapy must be lifelong. Commercially available foods and formulas are available without BCAA. Even if affected individuals follow a specialised diet, a risk of metabolic crisis still exists. Surgical management includes Liver transplant. It may be an effective treatment.

It will not reverse brain damage but it may arrest brain damage. However, considering dietary therapy is low risk and long term complications of liver transplant both have equally good outcome.

\section{CONCLUSION}

Intermittent MSUD is potentially a life threatening metabolic disorder and should be suspected in cases of common infections with a clinically atypical course, especially in children with ataxia or marked drowsiness. An important point is that affected children are asymptomatic between episodes and laboratory tests are normal.

\section{ABBREVIATIONS}

BCAA - Branched Chain Amino Acids. BCKD-Branched Chain Alpha Keto Acid dehydrogenase complex, MSUD-Maple Syrup Urine Disease

\section{REFERENCES}

1. Menkes JH, Hurst PL, Craig JM. A new syndrome: progressive familial infantile cerebral dysfunction associated with an unusual urinary substance. Pediatrics 1954;14(5):462-7.

2. Dancis J, Levits M, Westall RG. Maple syrup urine disease: branched-chain ketoaciduria. Pediatrics 1960;25:72-9.

3. Genetics home reference website. ghr.nlm.nih.gov.

4. Brismar J, Aqeel A, Brismar G, et al. Maple syrup urine disease. AJNR 1990;11(6):1219-28.

5. Harris RA, Joshi M, Jeoung $\mathrm{NH}$, et al. Overview of the molecular and biochemical basis of branched-chain amino acid catabolism. J Nutr 2005;135(6 Suppl):1527S30s.

6. Morton DH, Strauss KA, Robinson DL, et al. Diagnosis and treatment of maple syrup disease: a study of 36 patients. Pediatrics 2002;109(6):999-1008.

7. Hallam P, Lilburn M, Lee PJ. A new protein substitute for adolescent and adults with maple syrup urine disease (MSUD). J Inherit Metab Dis 2005;28(5):665-72. 